\title{
Diminished Dopamine: Timing, Neuroanatomy, or Drug History?
}

\author{
Kurt M. Fraser ${ }^{1}$ and Joshua L. Haight ${ }^{2}$ \\ ${ }^{1}$ Department of Psychological \& Brain Sciences, Johns Hopkins University, Baltimore, Maryland 21218, and ${ }^{2}$ Neuroscience Graduate Program, University of \\ Michigan, Ann Arbor, Michigan 48109 \\ Review of Saddoris et al.
}

Determining why a relatively small percentage of the many individuals who experiment with drugs of abuse go on to develop substance abuse disorders is an important area of investigation. Understanding the neurobiological and psychological factors underlying the transition from casual drug use to addiction is critical for developing effective treatments. Animal models have helped researchers identify the actions of drugs of abuse and the processes underlying addiction, particularly how drug-associated stimuli come to instigate drug seeking, promote use, and trigger relapse following abstinence (Robinson and Berridge, 1993).

Decades of research have indicated that most drugs of abuse influence the actions of the mesolimbic dopamine system within the nucleus accumbens (NAc). Dopamine facilitates reward learning and the attribution of motivational value to relevant stimuli in the environment (Flagel et al., 2011; Steinberg et al., 2013), a phenomenon known as incentive salience

Received March 5, 2016; revised March 25, 2016; accepted March 30, 2016. This work was supported by National Research Service Award Fellowship DA037680 to J.L.H. We thank Dr. Shelly Flagel and Dr. Patricia Janak as well as Ben Saunders, Jocelyn Richard, Brittany Kuhn, Aram Parsegian, Paolo Campus, and Chris Fitzpatrick for their continued support, comments, and discussions.

The authors declare no competing financial interests.

Correspondence should be addressed to Kurt M. Fraser, Department of Psychological \& Brain Sciences, Johns Hopkins University, 3400 North Charles Street, 224 Dunning Hall, Baltimore, MD 21218. E-mail: kfraser3@jhu.edu.

DOI:10.1523/JNEUROSCI.0731-16.2016

Copyright $\odot 2016$ the authors $\quad 0270-6474 / 16 / 364907-03 \$ 15.00 / 0$ attribution (Robinson and Berridge, 1993). There is remarkable individual variability in the propensity to attribute incentive salience to reward-paired stimuli. Consequently, those that attribute incentive salience are more likely to reinstate reward-seeking behaviors following presentation of a previously reward-paired stimulus, particularly drug-associated stimuli, making abstinence from drug use incredibly difficult (Saunders and Robinson, 2010).

In a recent issue of the Journal of $\mathrm{Neu}$ roscience, Saddoris et al. (2016) examined how exposure to cocaine alters individual variability in the attribution of incentive salience to reward cues, and the relationship between this phenomenon and dopamine transmission in the NAc. In this study, water-restricted rats were trained to lever-press for either intravenous cocaine or for delivery of water in a reward port over the course of $14 \mathrm{~d}$. Then, after 1 month of forced abstinence, rats learned a Pavlovian discrimination task in which the presentation of a light-tone cue predicted the delivery of a sucrose reward. After rats learned the Pavlovian task, carbon fiber microelectrodes were implanted in the NAc core or shell subregions, to enable near real-time detection of dopamine release using fast-scan cyclic voltammetry during the final session of the Pavlovian task. Importantly, this allowed the authors to directly compare the impact of cocaine history on real-time dopamine release in NAc core and shell during performance of the Pavlovian task.
Pavlovian conditioning is often used to screen for individual variation in the propensity to attribute incentive salience to reward-associated stimuli. Some individuals, called "sign-trackers," attribute incentive salience to cues (i.e., find them motivationally attractive) and will vigorously approach and interact with a cue (Hearst and Jenkins, 1974). Other individuals, termed "goal-trackers," will instead approach the site of reward delivery upon cue presentation; for these individuals, the cue is purely a predictive stimulus (Boakes, 1977). Notably, drug history can bias animals toward a sign-tracking response, even when they may have originally had a predisposition to goal-track (Doremus-Fitzwater and Spear, 2011; Palmatier et al., 2013; Srey et al., 2015). Consistent with these findings, Saddoris et al. (2016) showed that rats with a history of cocaine self-administration were more likely to sign-track to a Pavlovian cue, whereas rats that self-administered water primarily goal-tracked. These data suggest that cocaine experience increases the propensity to attribute incentive salience to reward-paired cues in the future, even for cues paired with rewards other than cocaine.

There are, however, important limitations to consider in the interpretation of these findings. Saddoris et al. (2016) did not identify rats as sign- and goal-trackers in a Pavlovian task before self-administration, which would have identified inherent sign- and goal-tracking tendencies. 
Only then could the experimenters have directly attributed changes in Pavlovian conditioned responses to the impact of cocaine history. Additionally, the authors did not test whether cocaine exposure itself is sufficient to produce these results, which is an important caveat for two reasons. First, it is unclear whether rats injected with cocaine, withoutself-administration experience, would also be more prone to attribute incentive salience during later Pavlovian tasks. The inclusion of a yoked-cocaine control, a group that received the same pattern of cocaine infusion without performing an instrumental action, would have helped clarify the author's interpretations and elucidate whether these findings are related to the self-administration experience or to the pharmacological actions of cocaine itself. Second, the shift toward sign-tracking behavior may have resulted from neural plasticity changes happening during the abstinence period, rather than effects of cocaine itself.

Previous work has shown that dopamine transmission in the NAc core is linked to, and necessary for, the acquisition and expression of sign-tracking behavior (Flagel et al., 2011; Saunders and Robinson, 2012). Similar to these findings, Saddoris et al. (2016) confirmed that cue presentation elicits a large dopamine efflux in the NAc core in control signtrackers but not control goal-tracking rats, the groups that self-administered water during the first part of the experiment. Interestingly, in contrast to control signtrackers, cocaine-experienced sign-trackers showed very little dopamine release in response to the cue but had an exaggerated response to reward delivery (Saddoris et al., 2016, their Fig. 8). The observed dopamine responses are perplexing as they are not easily explained by current models of learning. First, if the signals were a reward prediction error signal (i.e., the value of the reward is able to facilitate learning about the cue-reward relationship), we would expect increased responding to cue presentation across trials and days. However, these signals are observed when learning is not ongoing as animals are well trained on the task, suggesting that they do not serve as a reward prediction error. Second, a majority of cocaineexperienced rats developed a sign-tracking response, even though these animals did not exhibit dopamine release to cue presentation in the NAc core that is thought to be necessary for the development and performance of this response. This "uncoupling" of phasic dopamine release in the NAc from cue presentation in cocaine-experienced sign-trackers raises interesting possibilities about the role of dopamine in incentive salience attribution following cocaine experience. Cueevoked dopamine efflux in the NAc core begins to diminish with extended Pavlovian training, whereas sign-tracking behavior remains robust, raising the possibility that dopamine signaling may shift to different striatal regions over time (Clark et al., 2013). Because this standard dopamine signature was still observed in control sign-trackers, one of many possible interpretations of these results is that cocaine exposure accelerated the diminishment of NAc core dopamine signals in sign-trackers and shifted this signal to dorsal striatal regions. In the current study, dopamine release was measured during a single session late in Pavlovian training, after sign-tracking was stable. Thus, it is possible that experimenters missed the evolution of the typical NAc dopamine signature to cue presentation in cocaine-experienced sign-trackers. Indeed, Willuhn et al. (2012) observed a similar decrease in dopamine release in the NAc in response to drug-cue presentation following prolonged cocaine selfadministration, and a concomitant increase in drug-cue dopamine signaling in the dorsal striatum (Willuhn et al., 2012). However, with dopamine measurements occurring at only one time point in the current study, it is difficult to conclude the origins and implications of diminished dopamine release in response to the cue in the NAc core of sign-trackers following cocaine exposure.

Another finding meriting further discussion concerns the patterns of dopamine release observed in the NAc shell, as it follows a pattern separable from the NAc core. For control sign-trackers, cue presentation resulted in dopamine release that remained elevated throughout its presentation, which is in contrast to the brief dopamine release to cue presentation in the NAc core. Control goaltrackers exhibited low levels of dopamine release in the NAc shell to the cue, and dopamine release was highest for reward delivery, a pattern that resembled release in the NAc core (Saddoris et al., 2016, their Fig. 9). These results indicate that dopamine release in the NAc shell follows a pattern distinct from release in the NAc core during sign-tracking behavior in control rats. These findings raise an important question: what is encoded by NAc shell dopamine signaling? Evidence suggests that pharmacologically increasing dopamine in the NAc shell enhances the ability of reward-predictive cues to invigorate ongoing reward-seeking (Peciña and Berridge, 2013), suggesting that NAc shell dopamine encodes an incentive motivational signal. This hypothesis is supported by the current findings, which show elevated NAc shell dopamine levels throughout the entire cue period for signtrackers only. If NAc shell dopamine is related to the incentive motivational properties of reward cues, then one would expect the signal to persist and be prevalent in drug-experienced sign-trackers. However, neither sign- nor goal-trackers with a history of cocaine experience exhibited dopamine release in the NAc shell during either cue presentation or reward delivery. This phenomenon is hard to interpret because the characteristics of dopamine in the NAc shell during the acquisition and ongoing expression of sign- and goal-tracking behavior are almost completely unknown. Determining the functional implications of dopamine signaling in NAc shell, and the significance of its loss following cocaine experience, remains an important question.

In conclusion, the results of Saddoris et al. (2016) highlight alterations in phasic dopamine release events in the NAc to reward-paired cues following cocaine exposure. Future research is needed to determine whether these signals are indeed aberrant or pathological, primarily through a better understanding of dopamine's function in normal conditions. Interestingly, the development of a sign- or goal-tracking phenotype promotes differing adaptations throughout the mesolimbic dopamine system, most prominently in the NAc (Flagel et al., 2007; Singer et al., 2015). The mechanisms underlying these adaptations remain to be elucidated. In addition, the possibility that differences in glutamatergic signaling converging on the nucleus accumbens, such as inputs from the amygdala, prefrontal cortex, and paraventricular thalamus, mediate these individual differences in rewardrelated behaviors should be explored. The study of individual differences in the attribution of motivational value to rewardassociated stimuli and its intersection with sophisticated models of psychiatric disorders is ripe for exploration, and we are enthusiastic for future developments made possible by this work.

\section{References}

Boakes R (1977) Performance on learning to associate a stimulus with positive reinforcement. In: Operant-Pavlovian interactions (Davis H, Hurwitz H, eds), pp 67-97. Hillsdale, NJ: Lawrence Erlbaum. 
Clark JJ, Collins AL, Sanford CA, Phillips PE (2013) Dopamine encoding of Pavlovian incentive stimuli diminishes with extended training. J Neurosci 33:3526-3532. CrossRef Medline

Doremus-FitzwaterTL,SpearLP (2011) Amphetamine-induced incentive sensitization of sign-tracking behavior in adolescent and adult female rats. Behav Neurosci 125:661-667. CrossRef Medline

Flagel SB, Watson SJ, Robinson TE, Akil H (2007) Individual differences in the propensity to approach signals vs goals promote different adaptations in the dopamine system of rats. Psychopharmacology (Berl) 191:599607. CrossRef Medline

Flagel SB, Clark JJ, Robinson TE, Mayo L, Czuj A, Willuhn I, Akers CA, Clinton SM, Phillips PE, Akil H (2011) A selective role for dopamine in stimulus-reward learning. Nature 469:5357. CrossRef Medline

Hearst E, Jenkins HM (1974) Sign-tracking: the stimulus-reinforcer relation and directed action. Austin, TX: Proceedings of the Psychonomic Society.

Palmatier MI, Marks KR, Jones SA, Freeman KS,
Wissman KM, Sheppard AB (2013) The effect of nicotine on sign-tracking and goaltracking in a Pavlovian conditioned approach paradigm in rats. Psychopharmacology (Berl) 226:247-259. CrossRef Medline

Peciña S, Berridge KC (2013) Dopamine or opioid stimulation of nucleus accumbens similarly amplify cue-triggered "wanting" for reward: entire core and medial shell mapped as substrates for PIT enhancement. Eur J Neurosci 37:1529-1540. CrossRef Medline

Robinson TE, Berridge KC (1993) The neural basis of drug craving: an incentive-sensitization theory of addiction. Brain Res Brain Res Rev 18:247-291. CrossRef Medline

Saddoris MP, Wang X, Sugam JA, Carelli RM (2016) Cocaine self-administration experience induces pathological phasic accumbens dopamine signals and abnormal incentive behaviors in drug-abstinent rats. J Neurosci 36: 235-250. CrossRef Medline

Saunders BT, Robinson TE (2010) A cocaine cue acts as an incentive stimulus in some but not others: implications for addiction. Biol Psychiatry 67:730-736. CrossRef Medline

Saunders BT, Robinson TE (2012) The role of dopamine in the accumbens core in the expression of Pavlovian-conditioned responses. Eur J Neurosci 36:2521-2532. CrossRef Medline

Singer BF, Guptaroy B, Austin CJ, Wohl I, Lovic V, Seiler JL, Vaughan RA, Gnegy ME, Robinson TE, Aragona BJ (2016) Individual variation in incentive salience attribution and accumbens dopamine transporter expression and function. Eur J Neurosci 43:662-670. CrossRef Medline

Srey CS, Maddux JM, Chaudhri N (2015) The attribution of incentive salience to Pavlovian alcohol cues: a shift from goal-tracking to sign-tracking. Front Behav Neurosci 9:54. CrossRef Medline

Steinberg EE, Keiflin R, Boivin JR, Witten IB, Deisseroth K, Janak PH (2013) A causal link between prediction errors, dopamine neurons and learning. Nat Neurosci 16:966-973. CrossRef Medline

Willuhn I, Burgeno LM, Everitt BJ, Phillips PE (2012) Hierarchical recruitment of phasic dopamine signaling in the striatum during the progression of cocaine use. Proc Natl Acad Sci U S A 109:20703-20708. CrossRef Medline 